\title{
Are we approaching the end of pediatric culture-negative osteoarticular infections?
}

\author{
Dimitri Ceroni ${ }^{*}, 1$, Romain Dayer ${ }^{1} \&$ Christina Steiger ${ }^{1}$ \\ ${ }^{1}$ Service of Orthopedics, Department of Child \& Adolescent, University of Geneva Hospitals \& University of Geneva Faculty of \\ Medicine, Geneva, Switzerland \\ *Author for correspondence: Tel.: +41 22 372 9164; Fax: +41 22382 4783; dimitri.ceroni@hcuge.ch
}

\begin{abstract}
"Once all pathogens responsible for OAI have been recognized, one can expect that NGS will evolve toward a selective amplification of specific genomic regions of interest, prior to massive simultaneous parallel sequencing”
\end{abstract}

First draft submitted: 14 May 2019; Accepted for publication: 21 June 2019; Published online: 2 August 2019

Keywords: osteoarticular infection • culture negative • nucleic acid amplification assays $\bullet$ PCR assays • nextgeneration sequencing

Osteoarticular infection (OAI) remains a serious pediatric diagnosis, with implications for future bone development and articular function [1]. Determining the proper etiology of $\mathrm{OAI}$ is essential to confirm diagnoses, allowing appropriate antibiotic prescription [2] to improve patient outcome [3]. The prognostic value concerns epidemiological surveillance, as identifying the causative germ has public health and infection control ramifications. A range of pathogens has been identified, and until recently Staphylococcus aureus was considered the most prevalent microorganism of OAIs in the pediatric population [4-9]. This simplistic vision of the epidemiology of OAI in pediatric populations has impeded improvements in our understanding of possible causes and its epidemiology for a long time, subsequently resulting in a delay in diagnostic and therapeutic developments for OAIs. Understanding of the microbiological causes of OAI has advanced significantly in recent years, and the clinical and biological characteristics of OAI are currently considered to be closely related to a child's age and to the pathogen responsible. The immune and vaccination status, comorbidities, socioeconomic conditions, changes in patterns of immunomodulating diseases and the emergence of resistant bacteria are contributing factors that also need to be considered [3].

Classical culture methods have traditionally been the cornerstone for establishing the diagnosis of OAI in children, searching for bacteria, mycobacteria, fungi, and, in rare cases, viruses and parasites as the possible causative agent. Using these culture-based diagnostic methods, negative cultures were frequently observed in pediatric OAI even if appropriate samples had been obtained. In fact, 24-68\% of acute hematogenous osteomyelitis (AHO) and 21-70\% of septic arthritis remained culture-negative, despite accurate attempts to identify the causative pathogen [10-13]. In these culture-negative infections, treatment decisions were thus more complex, requiring empirical treatment without knowledge of the pathogen. Treatment was solely based on epidemiological studies of culture-positive infections. In spite of these limitations, cultures still confer advantages over nonculture-based direct detection methods. In fact, a microorganism growing in classical cultures is available for accurate antimicrobial susceptibility testing. Furthermore, any microorganism that is grown in and can be accurately isolated from a culture, gives an unquestionably reliable result; a positive culture is therefore considered to have an absolute specificity.

Over the last decades, new screening methods have been developed for detecting bacteria to improve infectious disease diagnostics; some assays target microbial antigens by immunochemical techniques, whereas others detect microbial nucleic acid sequences. Since the beginning of the 2000s, the ability to detect small quantities of bacterial nucleic acids by means of nucleic acid amplification assays (NAAAs) has provided clinicians with a powerful set of tools for detecting low levels of bacteriological agents in clinical samples. In recent years, the technology of NAAAs has grown exponentially, with continued improvements in efficiency and specificity, for example, with the development of species-specific primers as opposed to universal primers, which are less prone to contaminant 
amplification. By using these methods, either specific antigens or nucleic acid segments may be detected directly in clinical samples. Consequently, NAAAs have become essential because of the unavoidable limitations of most culture techniques (e.g., technical complexity, insufficient sensitivity with certain fastidious organisms, expense and lack of timeliness). Thus, these new screening methods are currently used as adjuncts and, in selected instances, as substitutes to classical culture methods for determining the etiology of infection. Introduction of these diagnostic techniques into clinical bacteriological laboratories resulted in drastic improvements in the ability of clinicians to accurately diagnose infectious diseases. Thus, large scale use of NAAAs has drastically improved the diagnosis of OAI, and thereby changed both, the contemporary bacteriological epidemiology and the incidence of pediatric OAI. In fact, most recent studies using NAAAs have provided robust evidence that Kingella kingae is the most common pathogen responsible for OAI in children less than 4 years of age [14,15] and have highlighted the necessity to use specific real-time quantitative PCR (qPCR) assays targeting $K$. kingae to detect these infections more efficiently [16].

On this matter, an innovative and interesting diagnostic approach has been described in the last few years concerning the diagnosis of $K$. kingae infection. It has been demonstrated that detection of $K$. kingae in the oropharynx of children with suspected OAIs aged between 6 and 48 months had a sensitivity of $90.5 \%$ for $K$. kingae OAI infection [17]. This indirect and alternative diagnostic method was particularly attractive to clarify difficult clinical situations such as infantile spondylodiscitis, subacute osteomyelitis, arthritis affecting small joints or tenosynovitis, and to prove that most of these were primarily caused by $K$. kingae. A subsequent prospective case-control study, conducted in both Switzerland and Canada, has recently validated this innovative diagnostic approach at a wider scale [18]. This novel diagnostic approach has also been applied as an alternative strategy for recognized epidemic cases in French and Israeli outbreaks of $K$. kingae disease $[19,20]$. Although this diagnostic method could be considered controversial, this approach bears only few limitations. Only in rare situations, patients are asymptomatic $K$. kingae carriers and OAI are caused by alternative agents or multiple microorganisms, making these cases sporadic exceptions. A technical limitation is the necessity to have adequate material to perform optimal extraction of bacterial DNA.

However, despite recent advancements and a trend toward increasing broad use of PCR assays [14], $>20 \%$ of OAI's cases remain without proper bacterial diagnosis. This observation convinces us that many pediatric culturenegative osteoarticular infections are caused by unrecognized fastidious microorganisms, for which no molecular primers are currently available, highlighting the need for further attempts at characterization [14].

Finally, recent advances in next generation sequencing (NGS) have built a strong foundation for the development of more efficient methods for identifying microorganisms. NGS represents the targeted sequencing of all genomes present in a clinical sample, and thus does not depend on pathogens growing in cultures. NGS is based on high-performance DNA sequencing technologies, procuring millions of DNA strands and thus reducing the need for traditional cloning methods used in other genome sequencing techniques. NGS method can thus arbitrarily amplify and detect all the microorganisms that are present in a clinical sample, allowing theoretical recognition of all pathogens. However, NGS bears the disadvantage of arbitrarily amplifying all nucleic acids, including also host nucleic acids. Therefore, millions of reads need to be scrutinized to recognize pathogens of interest, presenting the main hurdle that slowed up the implementation of this technology in microbiological laboratories. Furthermore, a large number of reagents are required for deep sequencing and an elaborate database is a prerequisite for accurately evaluating such extensive datasets. Once all pathogens responsible for OAI have been recognized, one can expect that NGS will evolve toward a selective amplification of specific genomic regions of interest, prior to massive simultaneous parallel sequencing. This selective sequencing will provide increased sensibility, improved specificity and faster identification of pathogens of interest; at the same time, it will permit to control the price since more samples could be tested in the same run [21].

Advances in molecular sequencing technologies have opened new perspectives in the field of OAI diagnoses. In a near future, we could legitimately expect that these modern technologies will improve our capacity to detect and subsequently treat children with OAI, especially those due to fastidious pathogens. We are optimistic that these techniques will rapidly become a part of the microbiological acumen hopefully without a tremendous price explosion.

\section{Financial \& competing interests disclosure}

The authors have no relevant affiliations or financial involvement with any organization or entity with a financial interest in or financial conflict with the subject matter or materials discussed in the manuscript. This includes employment, consultancies, honoraria, stock ownership or options, expert testimony, grants or patents received or pending, or royalties. 
No writing assistance was utilized in the production of this manuscript.

\section{References}

1. Gutierrez K. Bone and joint infection. In: Principles and Practice of Pediatric Infectious Disease (2nd Edition). Long SS PL, Prober CG (Eds.). Churchill Livingstone, PA, USA, 467-474 (2003).

2. Luhmann JD, Luhmann SJ. Etiology of septic arthritis in children: an update for the 1990s. Pediatr. Emerg. Care 15(1), 40-42 (1999).

3. Dodwell ER. Osteomyelitis and septic arthritis in children: current concepts. Curr. Opin. Pediatr. 25(1), 58-63 (2013).

4. Chen WL, Chang WN, Chen YS et al. Acute community-acquired osteoarticular infections in children: high incidence of concomitant bone and joint involvement. J. Microbiol. Immunol. Infect. 43(4), 332-338 (2010).

5. Christiansen P, Frederiksen B, Glazowski J et al. Epidemiologic, bacteriologic and long-term follow-up data of children with acute hematogenous osteomyelitis and septic arthritis: a ten-year review. J. Pediatr. Orthop. B 8(4), 302-305 (1999).

6. Çaksen H, Kürşat Öztürk M, Uzüm K et al. Septic arthritis in childhood. Pediatr. Int. 42(5), 534-540 (2000).

7. Goergens ED, McEvoy A, Watson M et al. Acute osteomyelitis and septic arthritis in children. J. Paediatr. Child Health 41(1-2), 59-62 (2005).

8. Blyth MJ, Kincaid R, Craigen MA et al. The changing epidemiology of acute ans subacute haematogenous osteomyelitis in children. $J$. Bone Joint Surg. Br. 83(1), 99-102 (2001).

9. Karwowska A, Davies HD, Jadavji T. Epidemiology and outcome of osteomyelitis in the era of sequential intravenous-oral therapy. Pediatr. Infect. Dis. J. 17(11), 1021-1026 (1998).

10. Jaberi FM, Shahcheraghi GH, Ahadzadeh M. Short-term intravenous antimicrobial treatment of acute hematogenous bone and joint infection in children: a prospective randomized trial. J. Pediatr. Orthop. 22, 317-320 (2002).

11. Jagodzinski NA, Kanwar R, Graham K et al. Prospective evaluation of a shortened regimen of treatment for acute osteomyelitis and septic arthritis in children. J. Pediatr. Orthop. 29, 518-525 (2009).

12. Floyed RL, Steele RW. Culture-negative osteomyelitis. Pediatr. Infect. Dis. J. 22, 731-736 (2003).

13. Lyon RM, Evanich JD. Culture-negative septic arthritis in children. J. Pediatr. Orthop. 19, 655-659 (1999).

14. Juchler C, Spyropoulou V, Wagner $\mathrm{N}$ et al. The contemporary bacteriologic epidemiology of osteoarticular infections in children in Switzerland. J. Pediatr. 194, 190.e1-196.e1 (2018).

15. Ilharreborde B, Bidet P, Lorrot $\mathrm{M}$ et al. New real-time PCR-based method for Kingella kingae DNA detection: application to samples collected from 89 children with acute arthritis. J. Clin. Microbiol. 47(6), 1837-1841 (2009).

16. Ceroni D, Cherkaoui A, Ferey $S$ et al. Kingella kingae osteoarticular infections in young children: clinical features and contribution of a new specific real-time PCR assay to the diagnosis. J. Pediatr. Orthop. 30(3), 301-304 (2010).

17. Ceroni D, Dubois-Ferrière V, Cherkaoui A et al. Detection of Kingella kingae osteoarticular infections in children by oropharyngeal Swab PCR. Pediatrics 131, 1-6 (2013)

18. Gravel J, Ceroni D, Lacroix LE et al. Association between oropharyngeal carriage of Kingella kingae and osteoarticular infection in young children: a case-control study. Can. Med. Assoc. J. 189(35), E1107-E1111 (2017).

19. Yagupsky P, El Houmami N, Fournier PE. Outbreaks of Invasive Kingella kingae infections in daycare facilities: approach to investigation and management. J. Pediatr. 182, 14-20 (2017).

20. El Houmami N, Bzdrenga J, Pons JC et al. A modified multilocus sequence typing protocol to genotype Kingella kingae from oropharyngeal swabs without bacterial isolation. BMC Microbiol. 17(1), 200 (2017).

21. Dong L, Wang W, Li A et al. Clinical next generation sequencing for precision medicine in cancer. Curr. Genomics 16(4), 253-263 (2015). 
